This is an electronic reprint of the original article. This reprint may differ from the original in pagination and typographic detail.

Author(s): Sadhukhan, J.; Mazurek, K.; Dobaczewski, Jacek; Nazarewicz, W.; Sheikh, J.A.; Baran, A.

Title: Multidimensional Skyrme-density-functional Study of the Spontaneous Fission of $238 \mathrm{U}$

Year: $\quad 2015$

Version:

Please cite the original version:

Sadhukhan, J., Mazurek, K., Dobaczewski, J., Nazarewicz, W., Sheikh, J.A., \& Baran, A. (2015). Multidimensional Skyrme-density-functional Study of the Spontaneous Fission of 238U. Acta Physica Polonica B, 46(3), 575-578.

https://doi.org/10.5506/APhysPolB.46.575

All material supplied via JYX is protected by copyright and other intellectual property rights, and duplication or sale of all or part of any of the repository collections is not permitted, except that material may be duplicated by you for your research use or educational purposes in electronic or print form. You must obtain permission for any other use. Electronic or print copies may not be offered, whether for sale or otherwise to anyone who is not an authorised user. 


\title{
MULTIDIMENSIONAL SKYRME-DENSITY-FUNCTIONAL STUDY OF THE SPONTANEOUS FISSION OF ${ }^{238} \mathrm{U}^{*}$
}

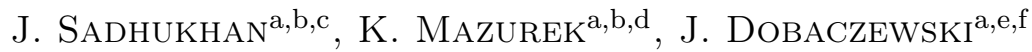 \\ W. NAzarewicz ${ }^{\mathrm{b}, e, \mathrm{~g}}$, J.A. Sheikh ${ }^{\mathrm{a}, \mathrm{b}}$, A. Baran $^{\mathrm{h}}$ \\ a Department of Physics and Astronomy, University of Tennessee \\ Knoxville, Tennessee 37996, USA \\ ${ }^{b}$ Physics Division, Oak Ridge National Laboratory \\ P.O. Box 2008, Oak Ridge, Tennessee 37831, USA \\ ${ }^{\mathrm{c}}$ Physics Group, Variable Energy Cyclotron Centre \\ 1/AF Bidhan Nagar, Kolkata 700064, India \\ dThe Henryk Niewodniczański Institute of Nuclear Physics, PAN \\ Radzikowskiego 152, 31-342 Kraków, Poland \\ eInstitute of Theoretical Physics, Faculty of Physics, University of Warsaw \\ Pasteura 5, 02-093 Warszawa, Poland \\ ${ }^{\mathrm{f}}$ Department of Physics, University of Jyväskylä \\ P.O. Box 35 (YFL), 40014 University of Jyväskylä, Finland \\ 'Department of Physics and Astronomy and NSCL/FRIB Laboratory \\ Michigan State University, East Lansing, Michigan 48824, USA \\ ${ }^{\mathrm{h}}$ Institute of Physics, University of M. Curie-Skłodowska \\ Radziszewskiego 10, 20-031 Lublin, Poland
}

\section{(Received March 23, 2015)}

We determined the spontaneous fission lifetime of ${ }^{238} \mathrm{U}$ by a minimization of the action integral in a three-dimensional space of collective variables. Apart from the mass-distribution multipole moments $Q_{20}$ (elongation) and $Q_{30}$ (left-right asymmetry), we also considered the pairingfluctuation parameter $\lambda_{2}$ as a collective coordinate. The collective potential was obtained self-consistently using the Skyrme energy density functional SkM*. The inertia tensor was obtained within the nonperturbative cranking approximation to the adiabatic time-dependent Hartree-FockBogoliubov approach. The pairing-fluctuation parameter $\lambda_{2}$ allowed us to control the pairing gap along the fission path, which significantly changed the spontaneous fission lifetime.

DOI:10.5506/APhysPolB.46.575

PACS numbers: 24.75.+i, 25.85.Ca, 21.60.Jz, 27.90.+b

* Presented at the Zakopane Conference on Nuclear Physics "Extremes of the Nuclear Landscape", Zakopane, Poland, August 31-September 7, 2014. 
This study of spontaneous-fission lifetimes is based on the energy-densityfunctional (EDF) theory and relies on the collective potential and inertia determined within the adiabatic time-dependent Hartree-Fock-Bogoliubov (ATDHFB) approach. In practical calculations, we use the Skyrme EDF parametrization $\mathrm{SkM}^{*}$ [1] and density-dependent pairing. The methodology adopted in this work strictly follows Refs. [2-4].

The ATDHFB inertia is calculated as

$$
\mathcal{M}_{i j}^{C}=\frac{1}{2 \dot{q}_{i} \dot{q}_{j}} \sum_{\alpha \beta} \frac{\left(F_{\alpha \beta}^{i *} F_{\alpha \beta}^{j}+F_{\alpha \beta}^{i} F_{\alpha \beta}^{j *}\right)}{E_{\alpha}+E_{\beta}},
$$

where $\dot{q}_{i}$ and $\dot{q}_{j}$ represent time derivatives of the collective coordinates. The sum is evaluated over all quasiparticle states and $E_{\alpha}$ denotes the quasiparticle energy. Matrices $F^{i}$ are obtained from

$$
-F^{i *}=\left(B^{T} \frac{\partial \rho}{\partial q_{i}} A+B^{T} \frac{\partial \kappa}{\partial q_{i}} B-A^{T} \frac{\partial \kappa^{*}}{\partial q_{i}} A-A^{T} \frac{\partial \rho^{*}}{\partial q_{i}} B\right) \dot{q}_{i},
$$

where $A$ and $B$ are the Hartree-Fock-Bogoliubov (HFB) matrices, obtained self-consistently from the constrained HFB equations. The particle and pairing densities, $\rho$ and $\kappa$ respectively, are determined uniquely from $A$ and $B$.

The total Routhian is

$$
H_{\mathrm{HFB}}^{\prime}=\hat{H}_{\mathrm{HFB}}-\sum_{l=2,3} q_{l} \hat{Q}_{l 0}-\sum_{\tau=p, n}\left(\lambda_{\tau} \hat{N}_{\tau}-\lambda_{2 \tau}\left(\hat{N}_{\tau}^{2}-\left\langle N_{\tau}^{2}\right\rangle\right)\right),
$$

where $\hat{H}_{\mathrm{HFB}}$ is the HFB Hamiltonian, $\hat{Q}_{20}$ and $\hat{Q}_{30}$ are quadrupole and octupole moments, respectively, and $\hat{N}_{\tau}$ is particle-number operator. The terms associated with $\lambda_{2 \tau}$ modify the pairing correlations of the system [2,5] that can be assessed through the average pairing gaps

$$
\Delta_{\tau}=\frac{\operatorname{Tr}^{\prime} \hat{\Delta}^{\tau} \rho_{\tau}}{\operatorname{Tr} \rho_{\tau}}
$$

where $\hat{\Delta}^{\tau}$ is the pairing field and $\operatorname{Tr}^{\prime} A=\sum_{n} A_{n \bar{n}}$, with bar over $n$ indicating the time-reversed state.

Calculations presented in this work were performed in a three-dimensional (3D) collective space, where moments $Q_{20}$ and $Q_{30}$ control axial nuclear shapes and $\lambda_{2}=\lambda_{2 p}=\lambda_{2 n}$ allows for simultaneously changing proton and neutron pairing correlations. An early discussion of the effect of pairing fluctuations on fission dynamics was presented, for example, in Refs. [6, 7] 
(see Ref. [2] for a comprehensive list of references). Although the potential energy $V$ increases as the pairing gap deviates from the HFB value, the collective inertia behaves as $\sim 1 / \Delta^{2}$ and, therefore, the minimum-action path favors stronger pairing correlations [2].

In this contribution, we carry out a comparative study of ${ }^{238} \mathrm{U}$, assuming axial geometry. The role and importance of other degrees of freedom, such as triaxiality [2], will be discussed elsewhere.

Potential energy surfaces shown in Fig. 1 allow us to study competition between the deformation and pairing effects. It turns out that the pairing

a)

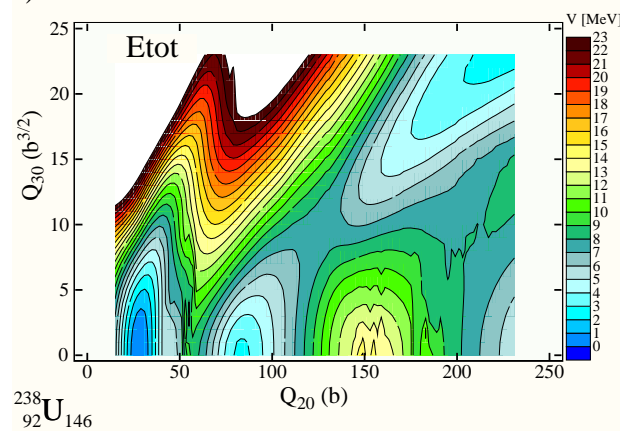

b)

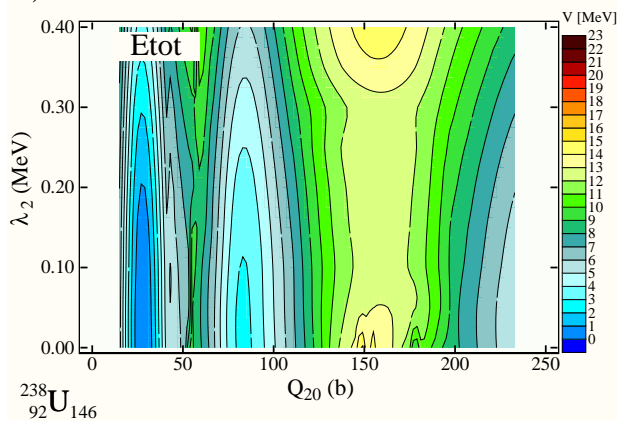

Fig. 1. Potential energy surfaces of ${ }^{238} \mathrm{U}$ in the $\left(Q_{20}-Q_{30}\right)$ plane for $\lambda_{2}=0.0$ (left) and in the $\left(Q_{20}-\lambda_{2}\right)$ plane for $Q_{30}=0.0$ (right).

fluctuations are more important around the first saddle than in the groundstate energy minimum. As it is shown in Fig. 2, with increasing pairing, the potential energy increases, whereas the mass tensor, in general, decreases. Such a competition significantly affects the fission lifetimes. For example, our $2 \mathrm{D}$ calculations (along the $\lambda_{2}=0$ path) yield $T_{\mathrm{SF}}=2.34 \times 10^{21} \mathrm{y}$, while the $3 \mathrm{D}$ calculations including pairing predict $T_{\mathrm{SF}}=3.63 \times 10^{17} \mathrm{y}$, which is closer to the experimental value of $8.2 \times 10^{15} \mathrm{y}$. This is consistent with findings of recent Refs. [8, 9] based on Gogny-EDF framework.

In summary, we performed a preliminary axial-symmetry study of spontaneous fission of ${ }^{238} \mathrm{U}$, in which pairing fluctuations were treated dynamically by minimizing the collective action. Using the microscopic input based on the ATDHFB approach, we obtained a fair agreement with experiment. 


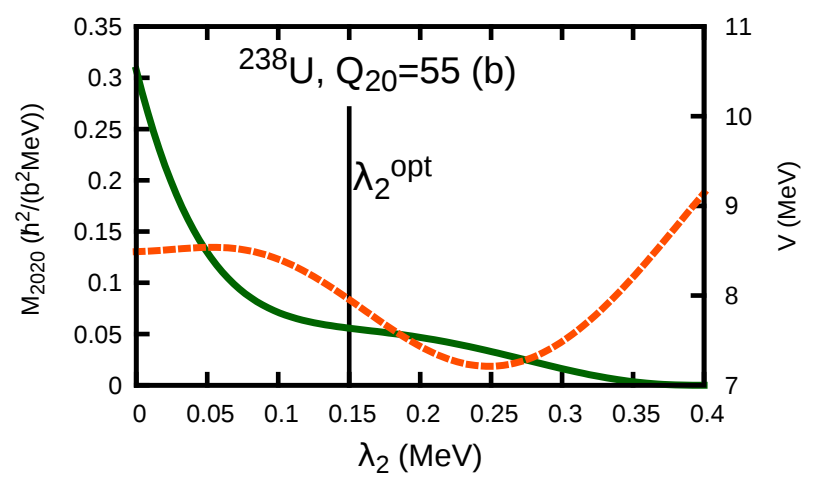

Fig. 2. The quadrupole diagonal inertia (solid line) and potential energy (dashed line) as functions of the pairing-fluctuation parameter $\lambda_{2}$. The multipole moments $\left(Q_{20}=55 \mathrm{~b}\right.$ and $\left.Q_{30}=0\right)$ correspond to the fission barrier. The vertical line marks the value of $\lambda_{2}^{\text {opt }}$ that corresponds to the calculated dynamical fission path.

This work was supported in part by the U.S. Department of Energy, Office of Science, Office of Nuclear Physics under Award Nos. DE-FG0296ER40963 (University of Tennessee) and DE-SC0008499 (NUCLEI SciDAC Collaboration); by the NNSA's Stewardship Science Academic Alliance Program under Award No. DE-FG52-09NA29461; by the Polish National Science Center under Contract No. 2012/07/B/ST2/03907; and by the Academy of Finland and University of Jyväskylä within the FIDIPRO programme.

\section{REFERENCES}

[1] J. Bartel et al., Nucl. Phys. A386, 79 (1982).

[2] J. Sadhukhan et al., Phys. Rev. C90, 061304 (2014).

[3] A. Baran et al., Phys. Rev. C84, 054321 (2011).

[4] J. Sadhukhan et al., Phys. Rev. C88, 064314 (2013).

[5] N.L. Vaquero et al., Phys. Lett. B704, 520 (2011); Phys. Rev. C88, 064311 (2013).

[6] L.G. Moretto, R.P.Babinet, Phys. Lett. B49, 147 (1974).

[7] A. Staszczak et al., Phys. Lett. B161, 227 (1985).

[8] R. Rodriguez-Guzman, L.M. Robledo, Phys. Rev. C89, 054310 (2014).

[9] S.A. Giuliani et al., Phys. Rev. C90, 054311 (2014). 